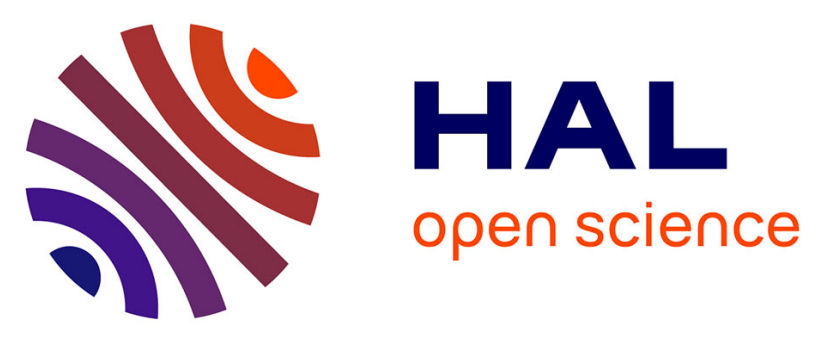

\title{
A Novel Image Analysis Methodology for the Evaluation of Angiogenesis in Matrigel Assays and Screening of Angiogenesis-Modulating Compounds
}

Ioannis Valavanis, Theodosios Goudas, Maria Michailidou, Ilias Maglogiannis, Heleni Loutrari, Aristotelis Chatziioannou

\section{To cite this version:}

Ioannis Valavanis, Theodosios Goudas, Maria Michailidou, Ilias Maglogiannis, Heleni Loutrari, et al.. A Novel Image Analysis Methodology for the Evaluation of Angiogenesis in Matrigel Assays and Screening of Angiogenesis-Modulating Compounds. 11th IFIP International Conference on Artificial Intelligence Applications and Innovations (AIAI 2015), Sep 2015, Bayonne, France. pp.61-71, 10.1007/978-3-319-23868-5_5 . hal-01385345

\section{HAL Id: hal-01385345 \\ https://inria.hal.science/hal-01385345}

Submitted on 21 Oct 2016

HAL is a multi-disciplinary open access archive for the deposit and dissemination of scientific research documents, whether they are published or not. The documents may come from teaching and research institutions in France or abroad, or from public or private research centers.
L'archive ouverte pluridisciplinaire HAL, est destinée au dépôt et à la diffusion de documents scientifiques de niveau recherche, publiés ou non, émanant des établissements d'enseignement et de recherche français ou étrangers, des laboratoires publics ou privés. 


\title{
A novel image analysis methodology for the evaluation of angiogenesis in matrigel assays and screening of angiogenesis-modulating compounds
}

\author{
Ioannis Valavanis ${ }^{1}$, Theodosios Goudas ${ }^{2}$, Maria Michailidou ${ }^{3}$, Ilias Maglogiannis ${ }^{2}$, \\ Heleni Loutrari ${ }^{3}$, and Aristotelis Chatziioannou ${ }^{1 *}$ \\ ${ }^{1}$ Institute of Biology, Medicinal Chemistry \& Biotechnology, National Hellenic Research \\ Foundation, Athens, Greece \\ ${ }^{2}$ University of Piraeus, Dept. of Digital Systems, Piraeus, Greece \\ ${ }^{3}$ G.P. Livanos and M. Simou Laboratories, $1^{\text {st }}$ Department of Critical Care Medicine \& \\ Pulmonary Services, Evangelismos Hospital, Medical School, University of Athens, Greece
}

*Corresponding author (achatzi@eie.gr)

\begin{abstract}
Angiogenesis, with a prominent role in tumor growth, is a physiological process through which new blood vessels are generated from preexisting vasculature. The effect of angiogenesis inhibition test agents to cell differentiation can be evaluated using the matrigel differentiation assay, an environment simulating in vitro the natural angiogenic process under study. Using this assay, angiogenic activity can be evaluated by the quantification of tubules, junctions and other structures of interest in the matrigel images, i.e. microphotographs of the formed cellular networks under different fields of view. In this work, we present an image analysis workflow that uses separate channels in the RGB matrigel images, each contributing different information related to angiogenic activity (tubules, junctions) and other structures (cells, lumens). The workflow is successfully showcased on the basis of a series of matrigel images generated for the evaluation of angiogenesis inhibition compounds, and results are cross-validated with a manual expert scoring.
\end{abstract}

Keywords: angiogenesis, tubules, matrigel, RGB image, image analysis

\section{Introduction}

Angiogenesis is a physiological complex process through which new blood vessels are generated from pre-existing vasculature. Endothelial cells, the building units of vasculature, form the internal lining of blood vessels. Endothelial cell invasion, migration, proliferation, differentiation and structural rearrangement are essential steps of the angiogenic process that are regulated by a balance between pro- and antiangiogenic factors. However under certain conditions, this balance is disrupted resulting in either poor vascularization, as in the case of ischemic disorders, or in abnormal vasculature, as in the case of tumors [1]. 
The prominent role of angiogenesis in tumor growth and metastasis was first described by Folkman in 1971 [2]. Since then, scientific literature has offered a massive information on the role of angiogenesis in the progression of several human diseases and new therapeutic strategies for their treatment have been proposed through targeting of the angiogenic process [3] [4].

One of the challenges researchers initially faced was the selection of scientifically and technically efficient methods for assessing the endothelial response to candidate angiogenesis inhibitors [5]. Ideally, an assay should be valid, methodologically straightforward, easily quantifiable, cost-effective and able to provide an environment simulating the natural angiogenic process under study. One of the assays that encases several of the afore-mentioned factors and has been extensively used is the capillarylike tube formation assay, otherwise known as matrigel differentiation assay. It is considered a suitable in vitro angiogenesis model as it examines the ability of endothelial cells to produce tubular structures when cultured on surfaces pre-coated with matrigel. It is a specialized commercially available polymer substance extracted from Engelbreth-Holm-Swarm murine sarcoma. Matrigel contains basement membrane components and mimics the conditions of the external tissue microenvironment of the endothelium [6]. This extracellular matrix activates differentiation pathways in endothelial cells enabling them to form networks that display tubular extensions of the cytoskeleton and dynamic rearrangements of the cells resembling vascular lumens. Microscopes are used to capture phase-contrast images of the angiogenesis environment in vitro [7]. The cells can be treated with test agents and their effects on endothelial cell differentiation are evaluated using quantification of tube number and length, manually or by image processing software techniques applied to the images obtained by the microscope [8].

Wimasis and Angiosys are two commercial software packages that are dedicated to angiogenesis quantification in matrigel images, each featuring certain specifications and capabilities [9]. In order that matrigel images are used by Angiosys, they need to be in prior processed (skeletonized) by a dedicated image-processing tool (e.g. Adobe Photoshop combined with an Image Processing Kit plug-in as described in [9]). Wimasis performs automatically the whole process of image processing and angiogenesis quantification. Most frequently extracted angiogenesis features include tubules length, number of tubules (separate paths in the angiogenic pathway), number of junctions (cross-points of tubules) and tubules area [9]. In [10], the red channel of matrigel image is used within the open source ImageJ software package to measure the area that cells cover in the image. A semi-automatic manual approach for measuring tubules and cells in matrigel images using the Scion Image, an image processing software freely available from the National Insitute of Health (NIH) website, is presented in [11].

In this work, we present a fully automatic workflow, written in Java and utilizing the ImageJ 1.47 library, for matrigel image processing and analysis, in order to quantify the angiogenesis presented in matrigel images and extract other useful structures in these images (cells, lumens). Differing from other approaches, we neatly use separate channels in the RGB matrigel images, each contributing different 
information related angiogenic activity (tubules, junctions) and other structures (cells, lumens) in order to maximize the efficiency of capturing the information content within the matrigel images. We showcase successfully the use of the workflow for the analysis of a series of matrigel images, used for the evaluation of angiogenesis inhibition activity of several compounds, and cross-validate results with a manual expert scoring. The rest of the paper is structured as follows: Section 2 presents matrigel images acquisition, along with sample images, and the image-processing workflow, while Section 3 presents and discusses the results.

\section{Materials and Methods}

In this Section, matrigel images and their formulation using the matrigel capillarylike tube formation assay are firstly described. Next, three image processing subworkflows, each detecting different structures in the matrigel images, are described.

\subsection{Matrigel capillary-like tube formation assay}

Matrigel capillary-like tube formation assay was performed as previously described in [12]. Briefly, serum-starved human umbilical cord vein endothelial cells (HUVEC) were cultured in 24-well plates pre-coated with matrigel in presence of test compounds SC1117, SC1119, SC1123 and SC1124 (0.2 - $2 \mu \mathrm{M})$. At the end point, cells were stained with Eosin-Haematoxylin and microphotographs, using a Leica microscope (20x magnification lens), of the newly formed cellular networks were obtained from 10 different fields of view [12]. Thus, a total of 10 matrigel images were extracted and analyzed per sample (each image was 2048x1532 pixels, 0.68 $\mu \mathrm{m} / \mathrm{pixel}$ ). Evaluation of the anti-angiogenic effects of the test compounds took place by image processing and analysis workflow described next, that measured various phenotypical characteristics of cellular differentiation such as number of cells, tubular length, cellular junctions where tubules may cross-point, and lumens (the inside of a tubular structure). Manual scoring of tubule number was also performed for crossvalidation of the image analysis data.

A sample matrigel image is presented in Fig. 1 (top). This image is the one chosen to showcase the results of the image processing analysis workflow in the next section.

\subsection{Matrigel Image Processing and Features Extraction}

Three image processing and analysis sub-workflows were implemented, each of which using a combination of popular image processing operators, processes a different channel in the RBG matrigel images and derives different features from the image. The image processing sub-workflows are described below.

- Blue Channel Processing for angiogenesis path and tubules' extraction: The workflow is presented in Fig. 2 and processes the blue channel containing the angiogenesis activity both in tubules and around/within junctions in order to segment the angiogenesis path. Image parts that are featured a closed contour of the angiogenesis path are characterized as lumens. Part of the angiogenic path beginning and closing with a junction or cell (see next workflows for 
their identification) is characterized as tubule. Furthermore, an automatic thresholding in the blue channel and the consequent calculation of the ratio of black to total image is used to derive an \% angiogenesis quantification in the image. Blue channel can be processed differently in order to identify the cells in the matrigel image, as well. The corresponding sub-workflow is presented in Fig. 3.

- Red Channel Processing for junctions' extraction: Collagen found in the junction can be clearly visible in the red channel in matrigel images and the sub-workflow used for this scope is presented in Fig. 4

The results of each of the workflows described above are merged in a finally resulting image, where tubules, junctions and cells are shown (see Fig. 1, bottom for the resulting image obtained from the original matrigel image presented in Fig. 1, top). Numerical results provided include quantification of angiogenesis (\%), number of lumens, number of cells, number of junctions, total length of angiogenesis path (number of pixels) and ratio of total area found in cells (\%).
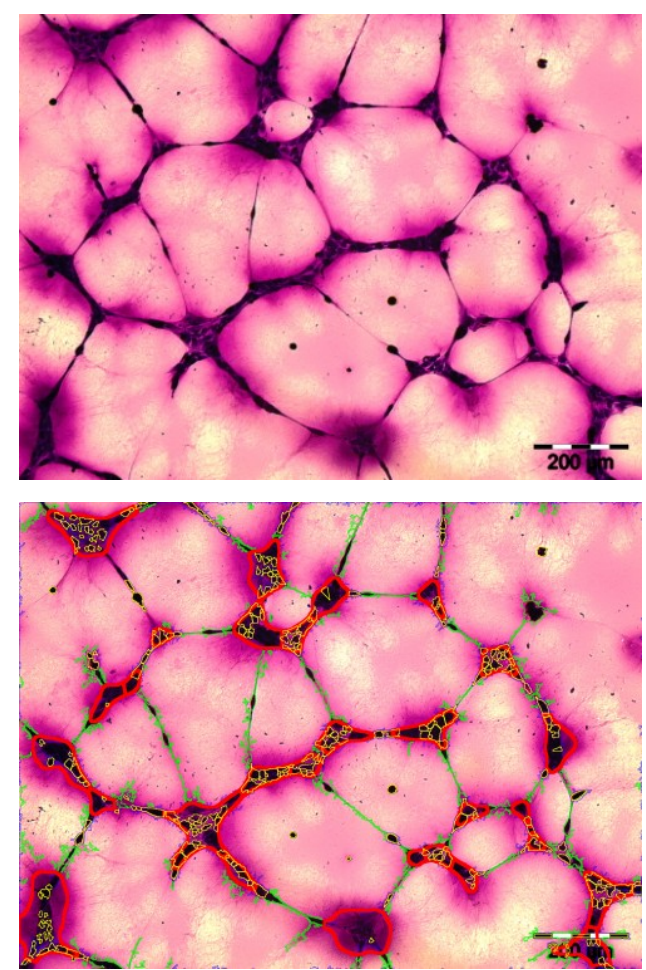

Fig. 1 Top: Original sample matrigel image. Bottom: Result of the image processing workflow (green corresponds to tubules, red color contours junction, and yellow contours cells) 

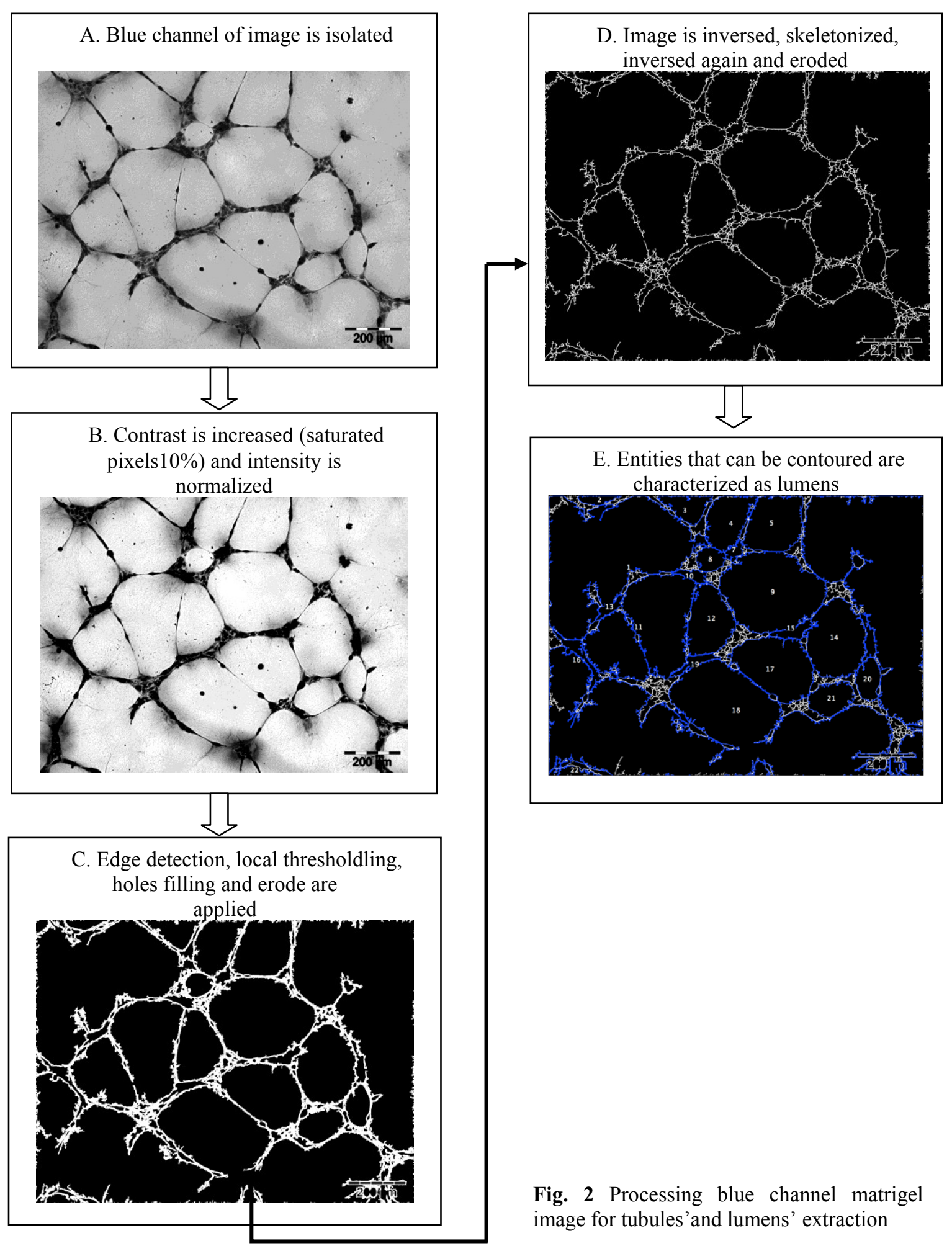

Fig. 2 Processing blue channel matrigel image for tubules'and lumens' extraction 

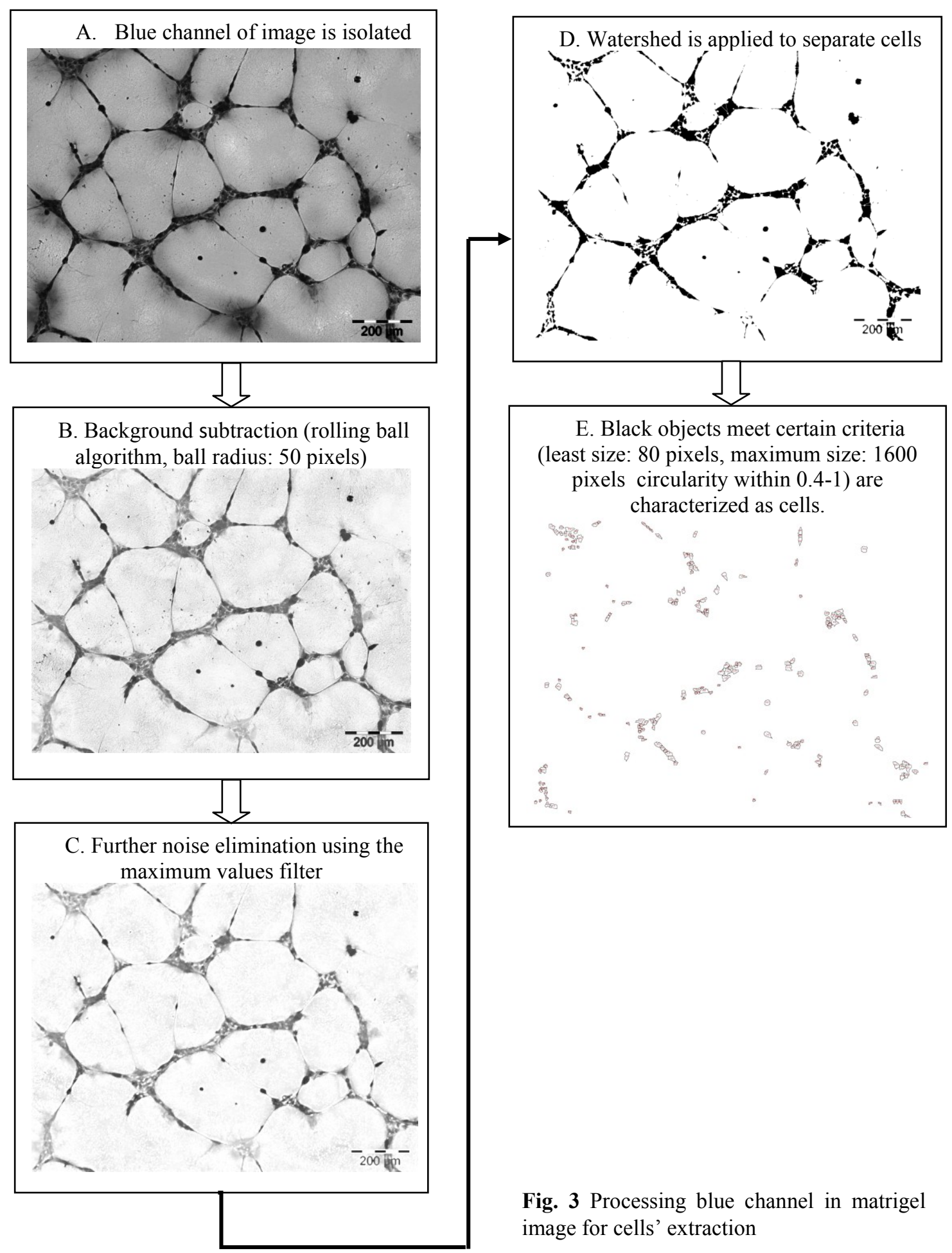

Fig. 3 Processing blue channel in matrigel image for cells' extraction 

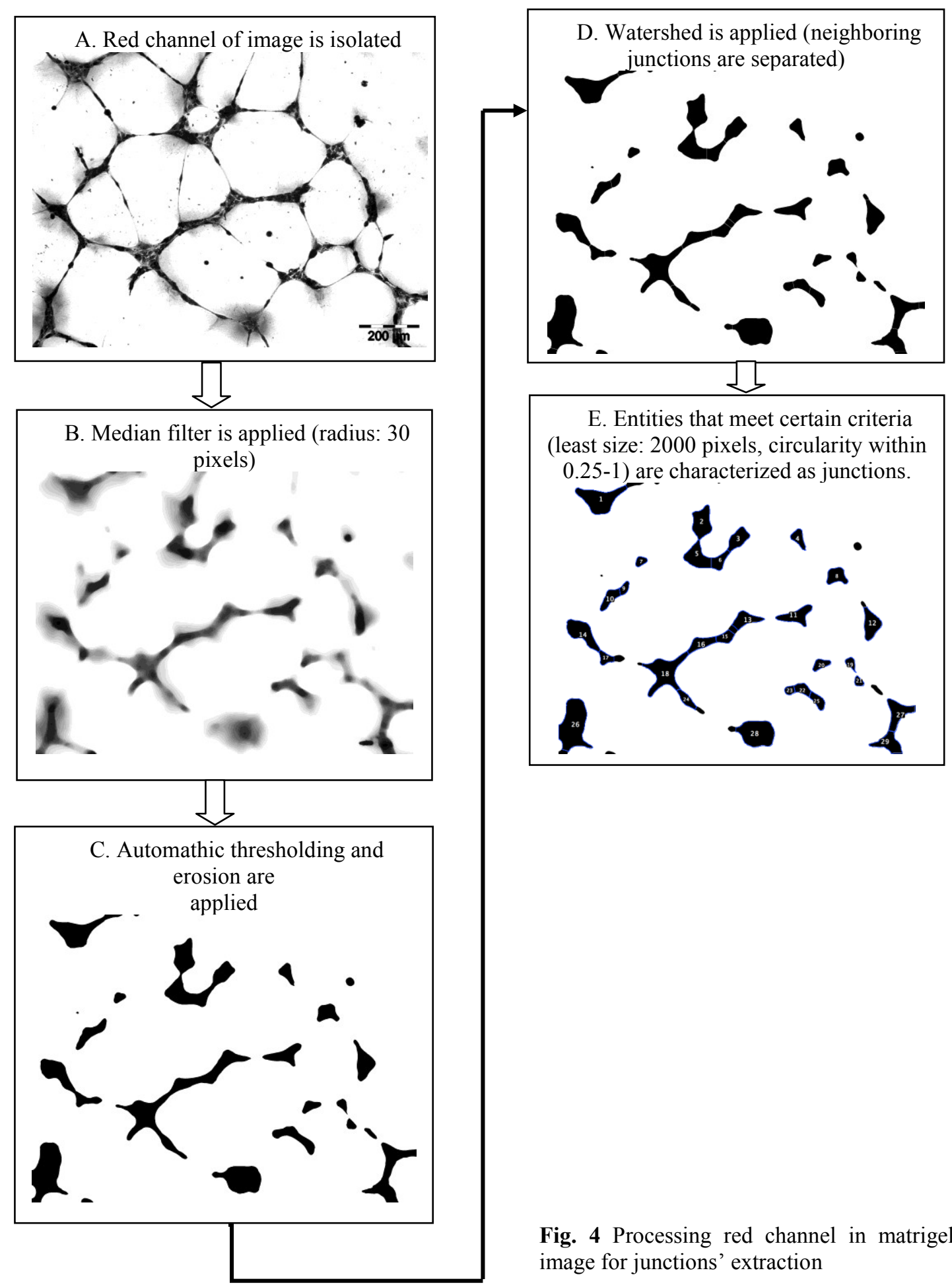

E. Entities that meet certain criteria (least size: 2000 pixels, circularity within $0.25-1)$ are characterized as junctions.

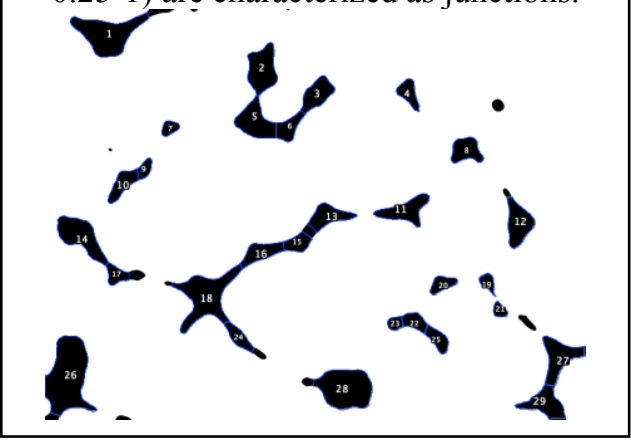

Fig. 4 Processing red channel in matrigel image for junctions' extraction 


\section{Results and Discussion}

The matrigel images extracted to evaluate the inhibition activity of the test

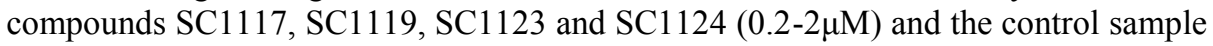
images (no inhibition compound applied) were analyzed. Each compound and the control sample were evaluated on the basis of the available images corresponding to the 10 fields of the formed cellular network. The workflow could real-time process the images and identify successfully structures of interest: tubules, cells, junctions and lumens. Sample imaging results are presented in Fig. 1 (bottom), where coloring is used to annotated the identified structures. Other imaging results not shown here support the efficiency of the algorithm to capture the structures of interest in the matrigel images.

Numerical results for each sample, calculated as average values of the features extracted for all images corresponding to the 10 fields of views, are presented in Table 1 (see Section 2.2 for description and extraction of the features used). A careful observation of the entries in Table 1 shows that the application of the inhibition compound reduces for most cases the angiogenic activity as measured by the features extracted that actually measure this activity, i.e. quantification of angiogenesis, total length of angiogenesis path, number of junctions. This appears more intensively for the increased concentration for the inhibition compounds $(0.2 \mu \mathrm{M}$ vs. $2 \mu \mathrm{M})$. Number of cells and lumens complete the angiogenesis information and can be used additionally by the expert.

Angiogenic activity reduction as measured by the quantification of angiogenesis $(\%)$ is presented graphically in Fig. 5, showing the effects of the various compounds and their concentration compared to the control sample. Manual scoring results for the evaluation of angiogenesis are presented in Fig. 6. Specifically, the tubule number in the matrigel images after the effects of the various compounds and their concentration, are presented as \% of tubule number measured in the control sample. Manual scoring results show the same pattern of angiogenesis reduction as implied by results regarding the automatic quantification of angiogenesis as presented in Fig. 5. Specifically, both manual and automatic analysis show that a greater concentration for the inhibition compounds results in a greater reduction in angiogenesis and that SC117 compound inhibits mostly the angiogenesis among all compounds tested.

Future work will include the evaluation and settings optimization of our image processing and analysis workflow using a larger dataset of images and the final evaluation of various natural or synthetic inhibition compounds on the basis of the matrigel capillary-like tube formation assay. A sophisticated analysis of the features extracted from a larger matrigel images dataset, will set the basis for a robust fully automated analysis workflow for the evaluation of endothelial response to candidate angiogenesis inhibitors. 
Table 1. Average values of features calculated for all 10 fields of views for each sample (control and five test compounds for concentrations $0.2 \mu \mathrm{M}$ and $2 \mu \mathrm{M}$ )

\begin{tabular}{|c|c|c|c|c|c|c|}
\hline Sample & $\begin{array}{l}\text { Quantification of } \\
\text { angiogenesis (x } 100 \\
\% \text { ) }\end{array}$ & $\begin{array}{c}\text { Number of } \\
\text { Junctions }\end{array}$ & $\begin{array}{l}\text { Number of } \\
\text { Cells }\end{array}$ & $\begin{array}{l}\text { Total length of } \\
\text { angiogenesis path } \\
\text { (pixels) }\end{array}$ & $\begin{array}{c}\text { Number } \\
\text { of Lumens }\end{array}$ & $\begin{array}{c}\text { Ratio of total } \\
\text { area found in cells }\end{array}$ \\
\hline Control & 0.096 & 21.769 & 71.692 & 34420 & 12.769 & 0.012 \\
\hline $\mathrm{SC} 1170.2 \mu \mathrm{M}$ & 0.062 & 14.909 & 79.455 & 31880 & 9.273 & 0.014 \\
\hline $\mathrm{SC} 1172 \mu \mathrm{M}$ & 0.056 & 9.600 & 92.200 & 30033 & 6.200 & 0.017 \\
\hline $\mathrm{SC} 1190.2 \mu \mathrm{M}$ & 0.091 & 25.000 & 100.600 & 34431 & 14.000 & 0.015 \\
\hline $\mathrm{SC} 1192 \mu \mathrm{M}$ & 0.062 & 12.500 & 124.500 & 32184 & 7.100 & 0.021 \\
\hline $\mathrm{SC} 1230.2 \mu \mathrm{M}$ & 0.072 & 20.100 & 87.900 & 35826 & 13.300 & 0.015 \\
\hline $\mathrm{SC} 1232 \mu \mathrm{M}$ & 0.070 & 18.818 & 91.000 & 34944 & 10.818 & 0.016 \\
\hline $\mathrm{SC} 1240.2 \mu \mathrm{M}$ & 0.071 & 21.800 & 59.500 & 30668 & 9.400 & 0.011 \\
\hline $\mathrm{SC} 1242 \mu \mathrm{M}$ & 0.063 & 16.700 & 64.400 & 33088 & 9.300 & 0.012 \\
\hline
\end{tabular}




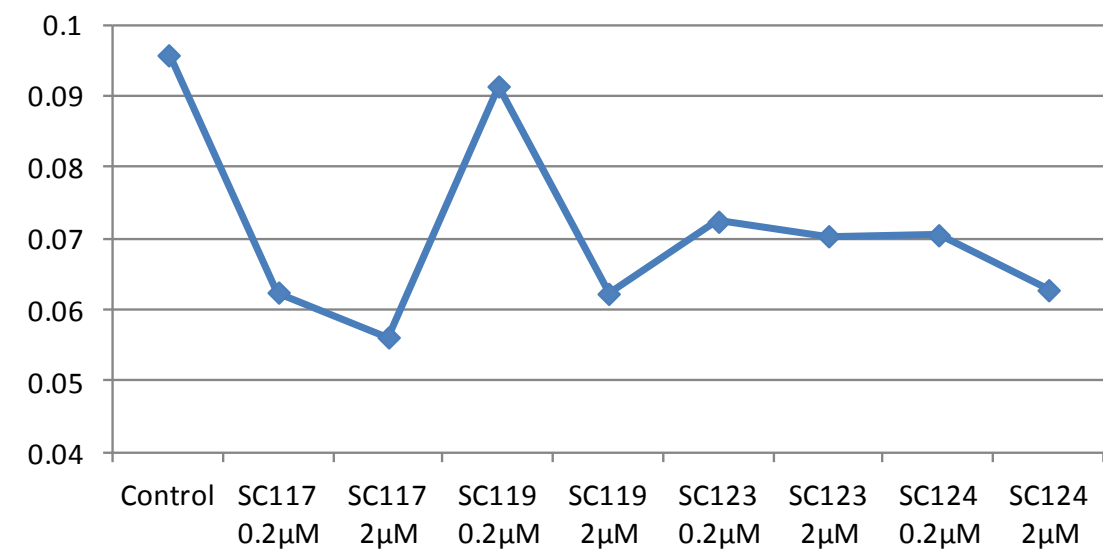

Fig. 5 Quantification of angiogenesis (x $100 \%$ ) showing the effects of the various compounds and their concentration compared to the control sample.

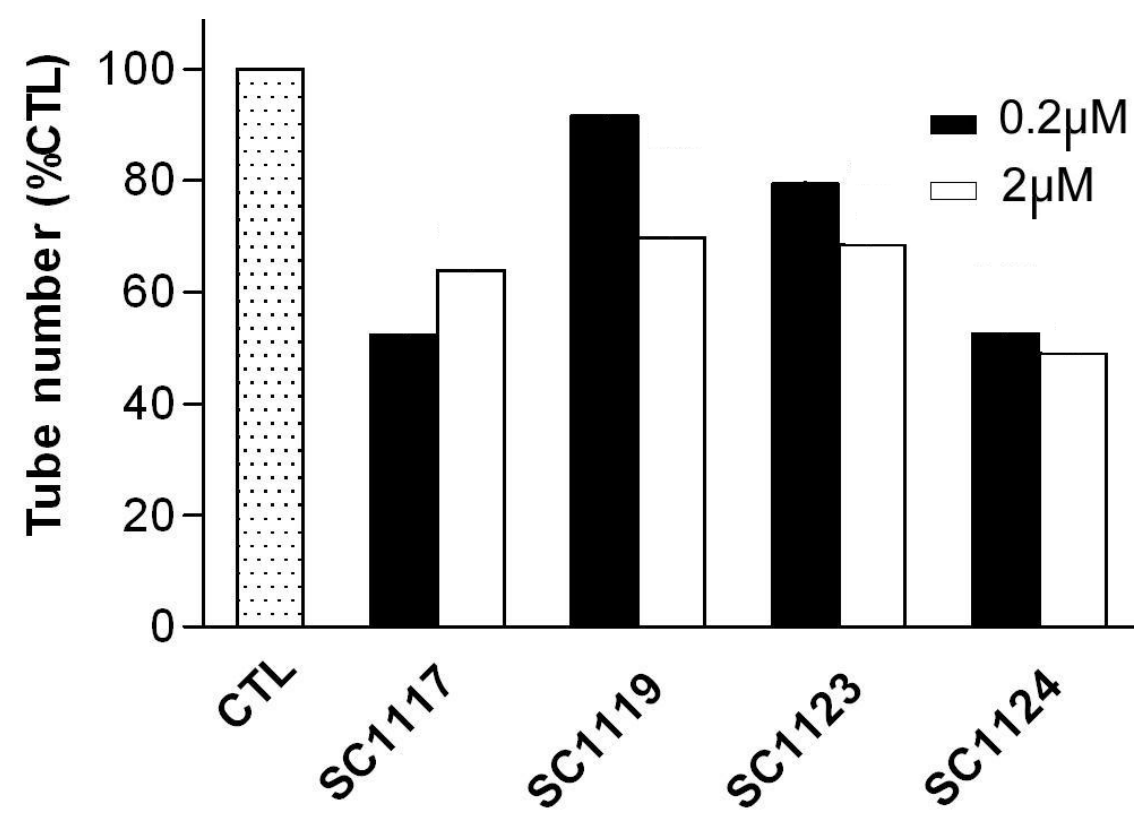

Fig. 6 Manual scoring results for the evaluation of angiogenesis (tubule number in the matrigel images after the effects of the various compounds are presented as \% of tubule number measured in the control sample) 


\section{Acknowledgement}

This work was supported by the Bilateral Greece-China Research Program of the Hellenic General Secretariat of Research and Technology and the Chinese Ministry of Research and Technology PROMISE (12CHN204, Personalization of melanoma therapeutic management through the fusion of systems biology and intelligent data mining methodologies).

\section{References}

[1] Potente M., Gerhardt H., Carmeliet P.: Basic and Therapeutic Aspects of Angiogenesis. Cell. 146 (6), 873-887 (2011)

[2] Folkman, J.: Isolation of a Tumor Factor Responsible for Angiogenesis. J. Exp. Med. 133, 275-288 (1971)

[3] Bridges, E.M., Harris, A.L.: The Angiogenic Process as a Therapeutic Target in Cancer. Biochem. Pharmacol. 81 (10), 1183-1191 (2011)

[4] Schmidt, T, Carmeliet, P. Angiogenesis: a target in solid tumors, also in leukemia? Hematology Am Soc Hematol Educ Program. (2011);2011:1-8. doi: 10.1182/asheducation-2011.1.1..

[5] El-Kenawi, A.E., El-Remessy, A.B., Angiogenesis inhibitors in cancer therapy: mechanistic perspective on classification and treatment rationales, $\mathrm{Br} J$ Pharmacol. (2013) Oct;170(4):712-29. doi: 10.1111/bph.12344..

[6] Hughes, C.S., Postovit, L.M., Lajoie, G.A. Matrigel: a complex protein mixture required for optimal growth of cell culture, Proteomics. (2010) May;10(9):1886-90. doi: 10.1002/pmic.200900758

[7] Ko, J.M. and Lung, M.L. (2012). In vitro Human Umbilical Vein Endothelial Cells (HUVEC) Tube-formation Assay. Bio-protocol 2(18): e260. http://www.bioprotocol.org/e260

[8] Doukas, C.N., Maglogiannis, I., Chatziioannou, A.A., Computer-supported angiogenesis quantification using image analysis and statistical averaging, IEEE Trans Inf Technol Biomed. (2008) Sep;12(5):650-7. doi: 10.1109/TITB.2008.926463. [9] Khoo, C.P., Micklem, K., Watt, S.M., A comparison of methods for quantifying angiogenesis in the Matrigel assay in vitro., Tissue Eng Part C Methods. (2011) Sep;17(9):895-906. doi: 10.1089/ten.TEC.2011.0150. Epub 2011 Jun 8., ,

[10] Gallo-Oller, G., Rey, J.A, Dotor, J., Castresana, J.S., Quantitative method for in vitro matrigel invasiveness measurement through image analysis software., Mol Biol Rep. (2014) Oct;41(10):6335-41. doi: 10.1007/s11033-014-3556-0. Epub 2014

[11] Donovan, D.,. Brown, N.J, Bishop, E.T, Lewis, C.E., Comparison of three in vitro human 'angiogenesis' assays with capillaries formed in vivo Angiogenesis (2001), Volume 4, Issue 2, pp 113-121,.

[12] Michailidou, M., Brown, H.K., Lefley, D.V., Evans, A., Cross, S.S., Coleman, R.E., Brown N.J., Holen, I.: Microvascular Endothelial Cell Responses in vitro and in vivo: Modulation by Zoledronic Acid and Paclitaxel? J. Vasc. Res. 47 (6), 481-493 (2010) 